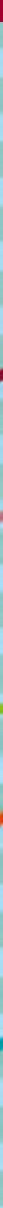

Unfallforschung kompakt

\title{
Verkehrsklima in Deutschland 2010: 10 Fragen zur Verkehrssicherheit
}

Unfallforschung der Versicherer 
Impressum

Gesamtverband der Deutschen Versicherungswirtschaft e. V.

Unfallforschung der Versicherer

Wilhelmstraße 43/43G, 10117 Berlin

Postfach 0802 64, 10002 Berlin

Unfallforschung@gdv.de

www.udv.de

Redaktion: Dr. rer. nat. Tina Gehlert

Layout: Franziska Gerson Pereira

Bildnachweis: Titelbild @SVLuma-Fotolia.com

Erschienen: 09/2011 


\section{Fragen zum Verkehrsklima in Deutschland}

Im Auftrag der Unfallforschung der Versicherer (UDV) führte das Marktforschungsinstitut TNS Infratest die Befragung „Verkehrsklima in Deutschland 2010“ durch. Die Befragung ist Teil der Längsschnittstudie "Verkehrsklima in Deutschland“, bei der im Zweijahresrhythmus die wahrgenommene Verkehrssicherheit und das Verkehrsverhalten der Bevölkerung erfasst werden. Die Ergebnisse werden auf der Webseite www.verkehrsklima.de veröffentlicht.

In die Befragung 2010 wurden vermehrt Fragen zum Radverkehr aufgenommen. Des Weiteren wurden neue Aspekte, wie das Fahren mit Licht am Tag erfragt. Schwerpunkt in der Befragung 2010 war die Einstellung und das Verkehrsverhalten von Geländewagen(SUV)-Fahrern. 


\section{Wie sicher fühlen sich die Deutschen im Straßenverkehr?}

Sicher oder sehr sicher im Straßenverkehr fühlen sich 53\% der Befragten, 12\% stufen ihr Sicherheitsempfinden als unsicher oder sehr unsicher ein und weitere $35 \%$ nehmen den Straßenverkehr als durchschnittlich sicher wahr. Im Vergleich zu 2008 ging die Anzahl der Personen, die sich im Straßenverkehr sehr sicher fühlen, um 16 Prozentpunkte von $69 \%$ auf 53 \% zurück. Des Weiteren gibt es einen Anstieg der Personen, die sich im Straßenverkehr nicht sicher fühlen von $6 \%$ auf 12\%. Die Mehrheit der Befragten fühlt sich aber auch 2010 sicher im Straßenverkehr.

\section{Wie sicher fühlen Sie sich im Straßenverkehr?}

Wahrgenommene

Verkehrssicherheit 2010

Wahrgenommene

Verkehrssicherheit 2008

$2010 \mathrm{n}=1.680$

$2008 n=1.002$

c) UDV 2010

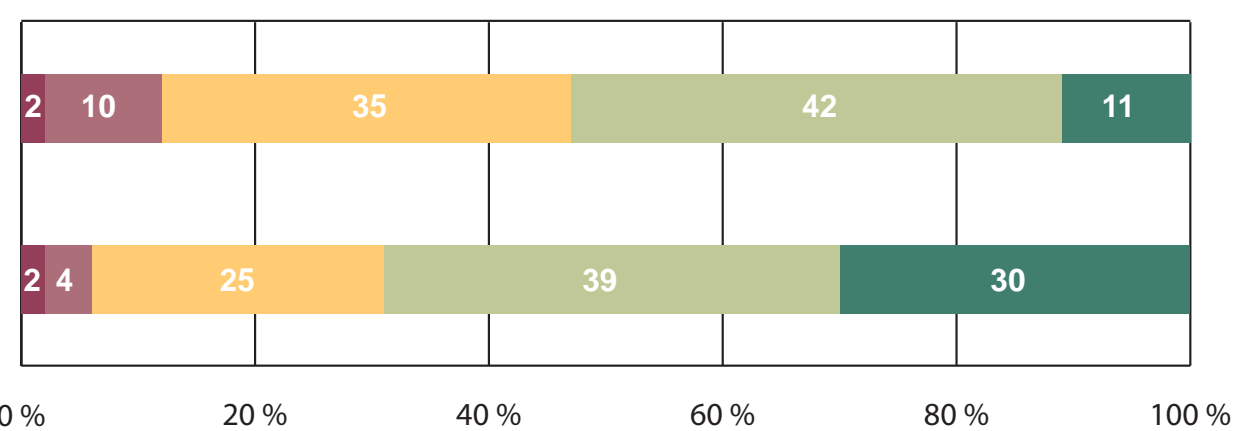

gar nicht sicher

sehr sicher

\section{Abbildung 1:}

Wie sicher fühlen Sie sich im Straßenverkehr?

Unterschiede in der wahrgenommenen Verkehrssicherheit gibt es zwischen den Verkehrsmitteln. $62 \%$ der Personen die fast täglich mit dem Pkw fahren, fühlen sich sicher oder sehr sicher im Straßenverkehr im Vergleich zu $55 \%$ der Personen, die fast täglich mit dem Fahrrad unterwegs sind, $52 \%$ der Personen, die fast täglich zu Fuß gehen oder mit dem ÖPNV fahren (48\%).

Tabelle 1:

Wahrgenommene Verkehrssicherheit nach Verkehrsmitteln

\begin{tabular}{|c|c|c|c|c|c|c|}
\hline Nutzergruppe & $\mathbf{N}$ & $\begin{array}{l}\text { Gar nicht } \\
\text { sicher }\end{array}$ & & & & $\begin{array}{c}\text { Sehr } \\
\text { sicher }\end{array}$ \\
\hline Fußgänger & 1.480 & $1,6 \%$ & $10,6 \%$ & $35,3 \%$ & $42,3 \%$ & $10,2 \%$ \\
\hline Pkw als Fahrer & 1.360 & $0,3 \%$ & $6,4 \%$ & $31,1 \%$ & $49,6 \%$ & $12,6 \%$ \\
\hline Fahrrad & 822 & $0,8 \%$ & $8,7 \%$ & $35,7 \%$ & $42,4 \%$ & $12,4 \%$ \\
\hline ÖPNV & 677 & $3,1 \%$ & $10,6 \%$ & $38,1 \%$ & $41,7 \%$ & $6,25 \%$ \\
\hline
\end{tabular}


Das Sicherheitsempfinden der Pkw-Fahrer nimmt ab, je weniger häufig sie den Pkw nutzen. So geben nur noch $25 \%$ der Personen an, die seltener als monatlich ein Auto fahren, ein sicheres oder sehr sicheres Gefühl im Straßenverkehr zu haben.

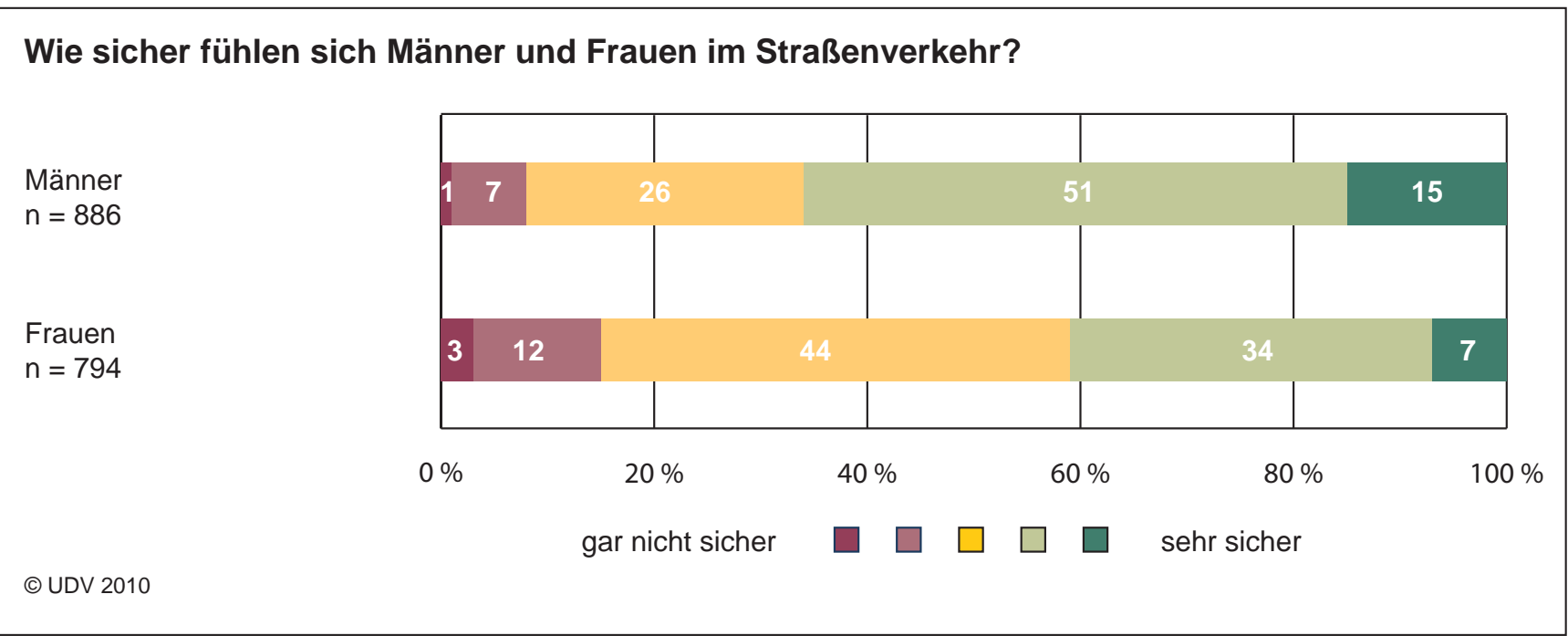

Abbildung 2:

Wie sicher fühlen sich Männer und Frauen im Straßenverkehr?

Frauen fühlen sich weniger sicher als Männer. $15 \%$ der Frauen führen an, sich im Straßenverkehr nicht sicher zu fühlen und etwa die Hälfte (44\%) empfindet den Verkehr auf den deutschen Straßen als nur durchschnittlich sicher. Im Vergleich stufen $65 \%$ der Männer den Verkehr als sicher oder sehr sicher ein. 


\section{Wo fühlen sich die Deutschen sicher?}

Am sichersten fühlen sich Pkw-Fahrer in Wohngebieten (73\%), gefolgt von der Landstraße (67\%) und innerstädtischen Hauptverkehrsstraßen (58\%). Autobahnen (57\%) und Straßen mit Baumalleen (49\%) werden von nahezu jedem zweiten Befragten als sicher oder sehr sicher eingestuft. Autobahnbaustellen empfindet lediglich jeder fünfte Pkw-Fahrer (20\%) als sicher. Frauen fühlen sich in Autobahnbaustellen unsicherer als Männer. So geben $16 \%$ der Frauen an, sich als PkwFahrer in Autobahnbaustellen sicher oder sehr sicher zu fühlen, im Vergleich zu $23 \%$ der Männer.

Diese Wahrnehmung steht im Widerspruch zu den bekannten Unfallzahlen. So weist die Unfallstatistik für Pkw-Fahrer für 2009 die wenigsten Unfälle mit Personenschaden und die niedrigste Anzahl an Verunglückten auf Autobahnen (18.394 Unfälle mit Personenschaden, 28.873 Verunglückte) im Vergleich zu Landstraßen (79.051 Unfälle mit Personenschaden, 114.031 Verunglückte) und Straßen innerhalb von Ortschaften aus (213.361 Unfälle mit Personenschaden, 258.919 Verunglückte). Ebenso gab es 2009 auf Autobahnbaustellen nur 1.191 Unfälle mit Personenschäden und 1.990 Verunglückte im Vergleich zu 17.203 Unfällen mit Personenschäden und 26.883 Verunglückten auf anderen Autobahnabschnitten.

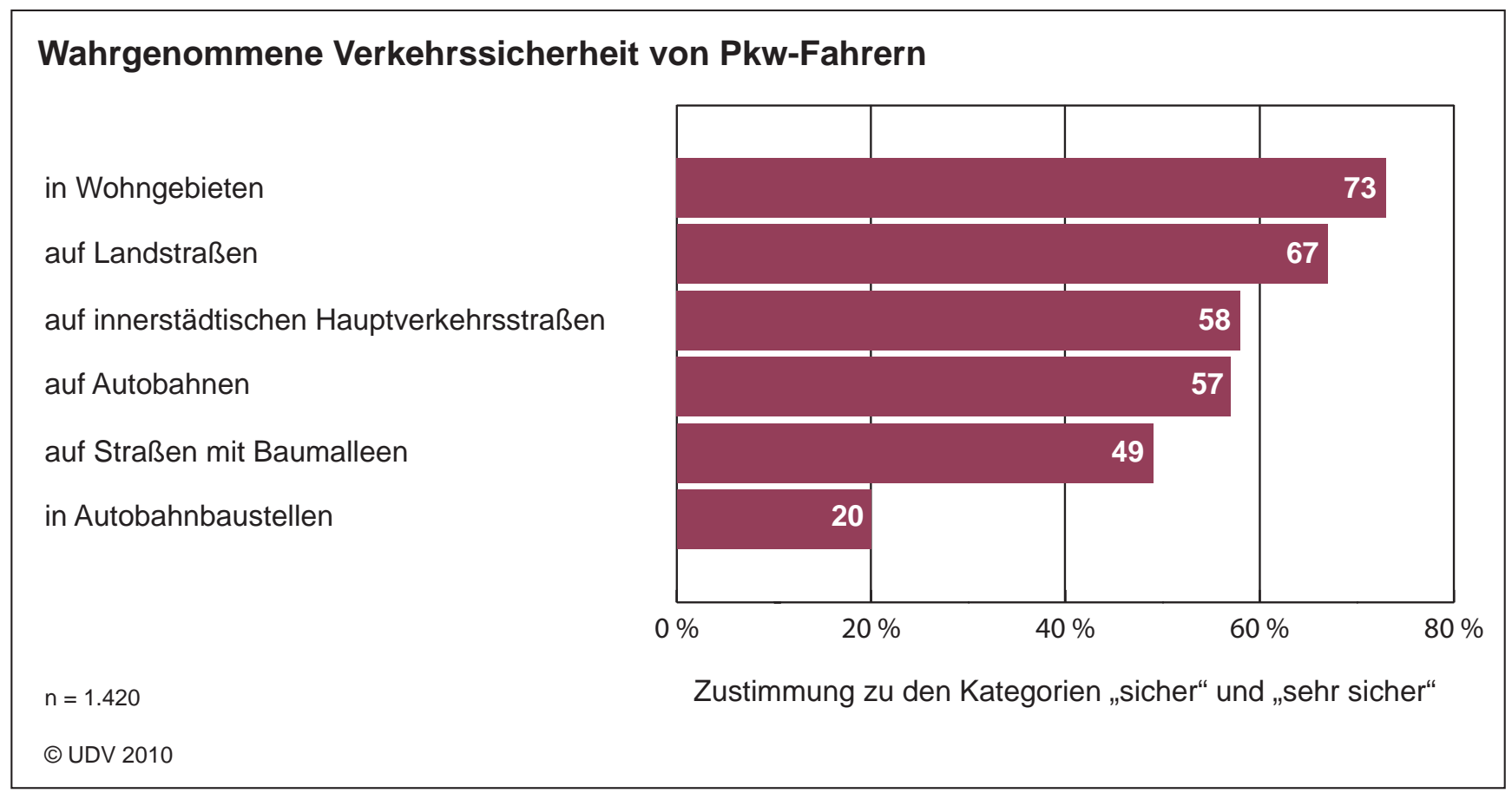

Abbildung 3:

Wahrgenommene Verkehrssicherheit von Pkw-Fahrern 
Als Fußgänger fühlen sich die Befragten am sichersten mit sogenannten Querungshilfen. Dabei wurde von fast $3 / 4$ der Fußgänger (73\%) die Fußgängerampel als sicherste Querungshilfe genannt. Vor allem ältere Menschen, ab einem Alter von 65 Jahren, fühlen sich an Fußgängerampeln sehr sicher. Weitere Querungshilfen (Zebrastreifen oder Mittelinseln) werden von fast jedem zweiten Befragten als sicher eingestuft. Im Gegensatz dazu steht eine Untersuchung der Unfallforschung aus dem Jahr 2006, die zeigt, dass jede Querungshilfe, sofern sie regelkonform gestaltet ist, die Sicherheit von Fußgängern gleichermaßen gewährleistet. Gemeinsame Fuß- und Radwege oder Straßen ohne jegliche Querungshilfen werden von den Befragten als am wenigsten sicher eingestuft.

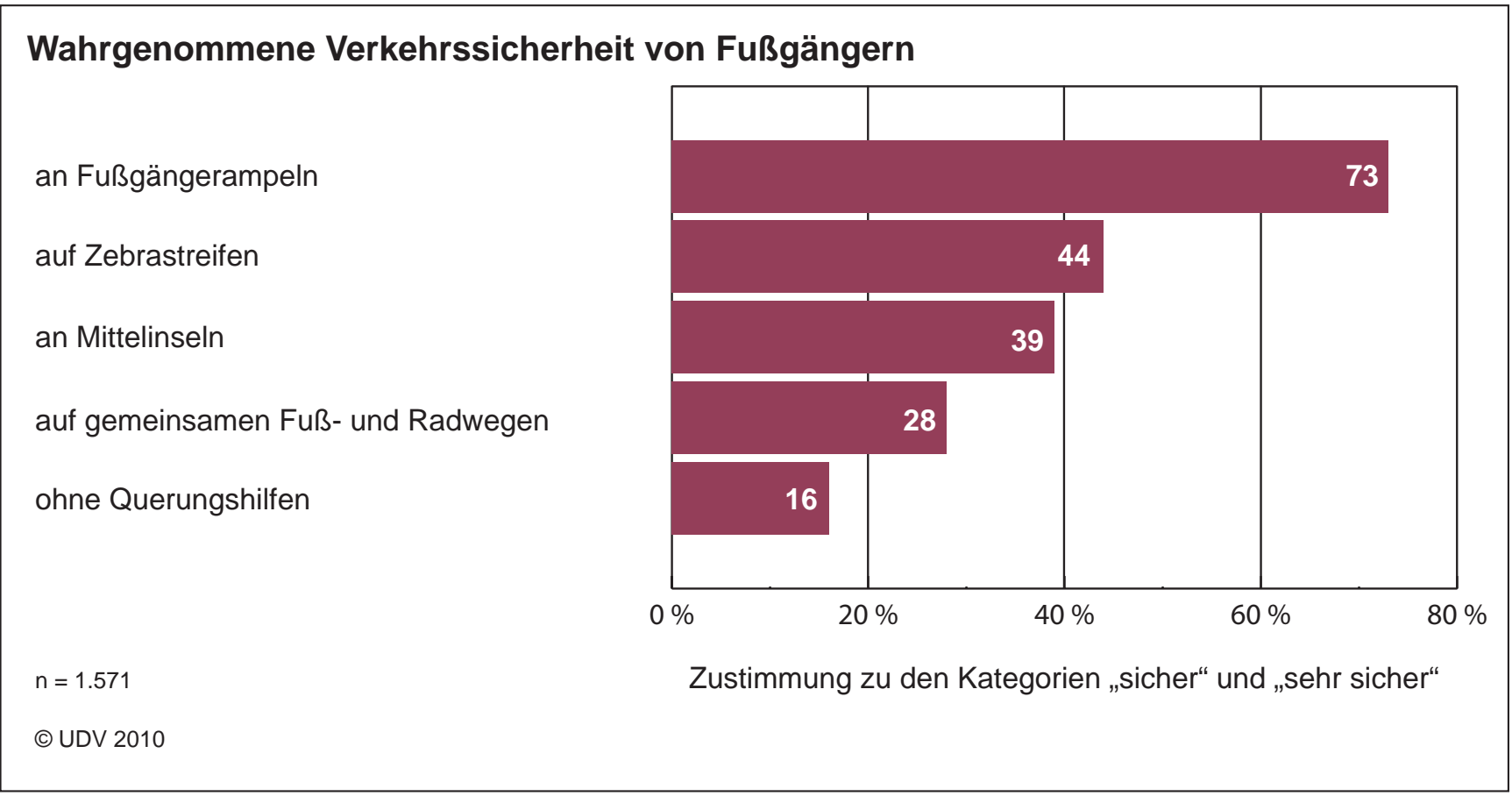

Abbildung 4:

Wahrgenommene Verkehrssicherheit von Fußgängern 
Der separate Radweg wird von $78 \%$ der Radfahrer als der sicherste Ort betrachtet. Der Fuß- und Radweg wird immerhin noch von jedem zweiten Befragten als sicher oder sehr sicher eingestuft. Damit schätzten etwa doppelt so viele Radfahrer wie Fußgänger den gemeinsamen Fuß- und Radweg als sicher oder sehr sicher ein.

Deutlich unsicherer wird die Nutzung der Fahrbahn betrachtet. Nur 14\% der Radfahrer fühlen sich beim Fahren auf der Fahrbahn auf einem gemeinsam mit dem Autoverkehr geführten Radstreifen sicher oder sehr sicher. Und nur 10\% der Radfahrer, unabhängig vom Geschlecht oder vom Alter, empfinden das Fahren auf der Fahrbahn ohne Radstreifen als sicher oder sehr sicher.

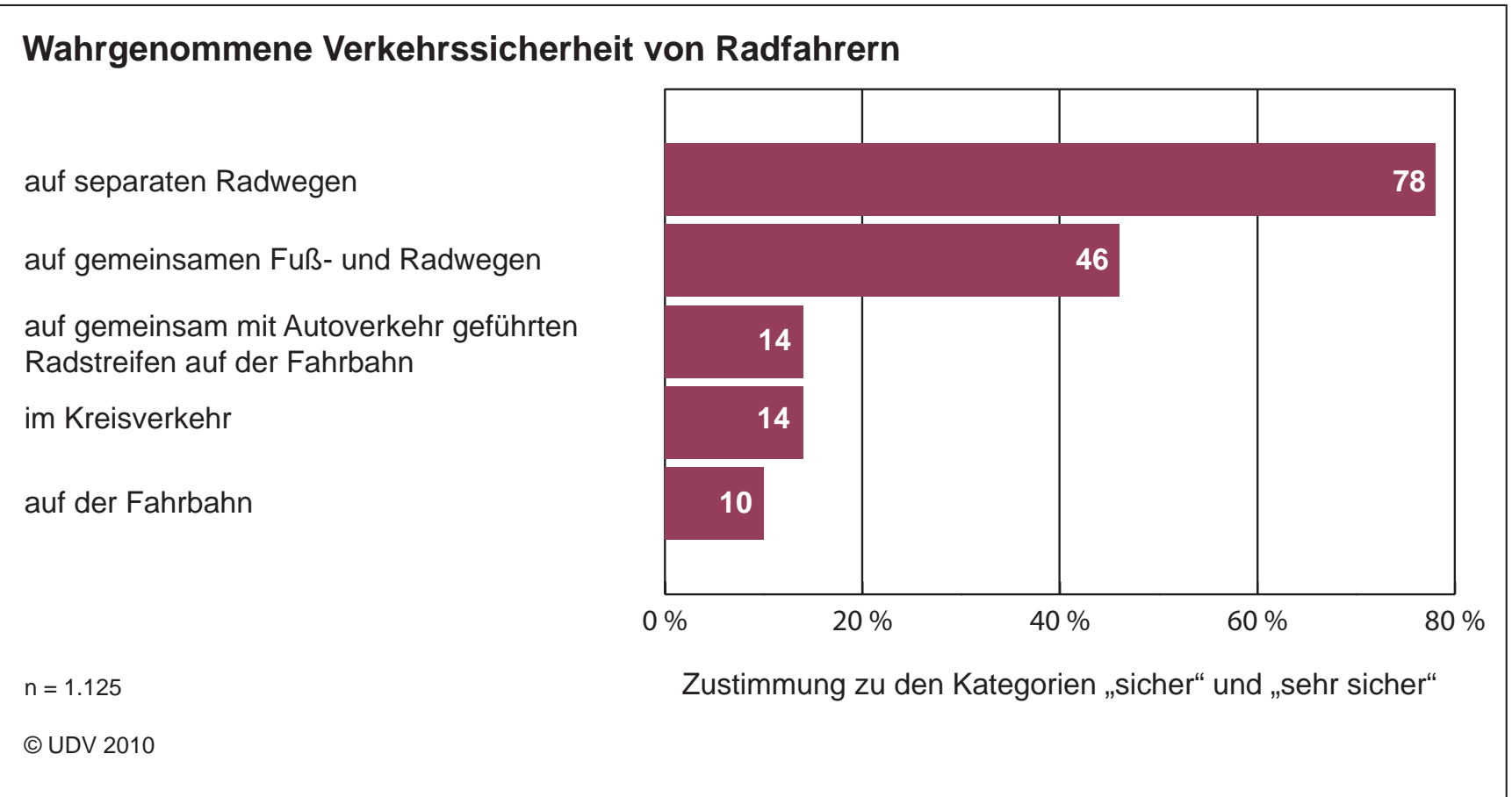

\section{Abbildung 5:}

Wahrgenommene Verkehrssicherheit von Radfahrern 


\section{Wer hat welches Risiko im Straßenverkehr?}

In den vorangegangenen drei Monaten erlebten 39\% der Befragten mindestens eine riskante Situation im Straßenverkehr bei der ein Unfall gerade noch vermieden werden konnte.

\section{Riskante Situationen im Straßenverkehr in den letzten drei Monaten}

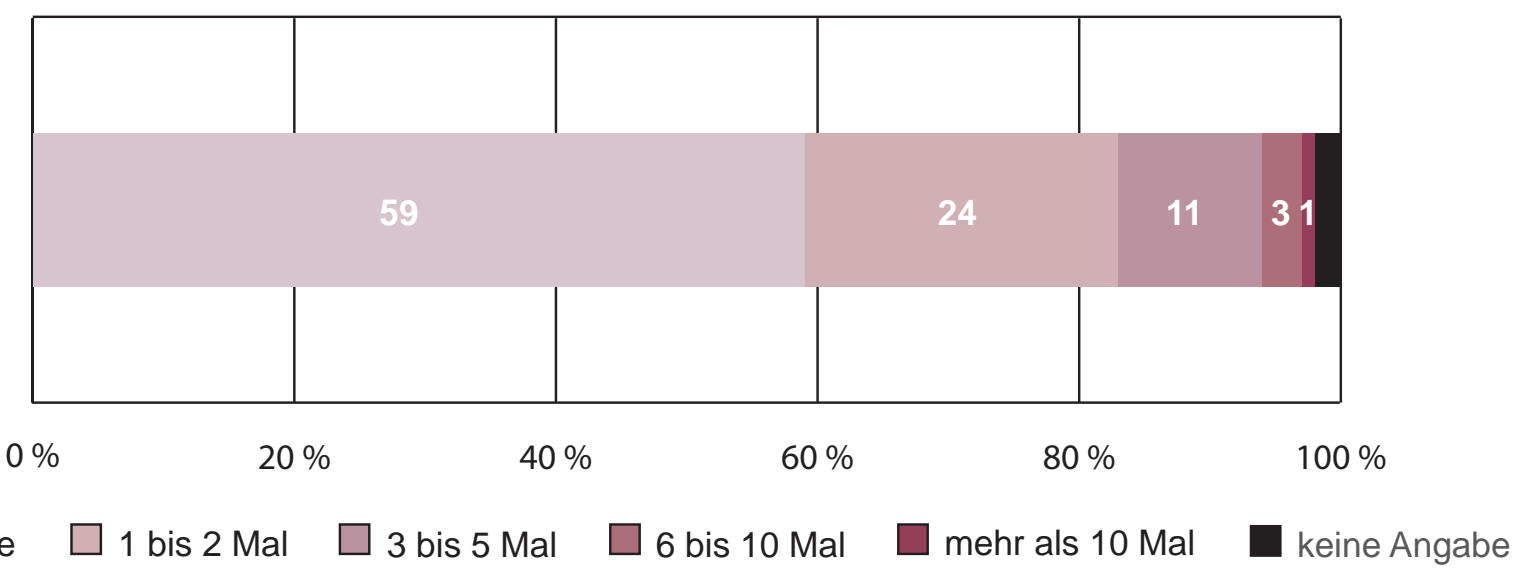

Basis: $\mathrm{n}=1.680$

(c) UDV 2010

Abbildung 6:

Riskante Situationen im Straßenverkehr

Besonders häufig erleben junge Verkehrsteilnehmer riskante Situationen im Straßenverkehr. Innerhalb der Altersgruppe 18 bis 24 Jahre gaben $63 \%$ der Befragten an, in den vorausgegangenen drei Monaten mindestens eine riskante Situation im Straßenverkehr erlebt zu haben, im Vergleich zu $48 \%$ innerhalb der Altersgruppe 25 bis 64 Jahre.

Neben jungen Verkehrsteilnehmern geraten auch Männer häufiger in riskante Situationen als Frauen. Nahezu jeder zweite männliche Pkw-Fahrer (44\%) erlebte mindestens eine riskante Situation in den vorangegangenen drei Monaten. Bei den Frauen waren es 36\%. 


\section{Wer sollte mehr für die Verkehrssicherheit in Deutschland tun?}

Die Mehrheit der Befragten (53\%) vertreten die Meinung, dass die anderen Verkehrsteilnehmer mehr für die Verkehrssicherheit in Deutschland tun müssen. Jedoch zeigt sich, dass die Hälfte der Befragten (51\%) auch sich selbst in der Verantwortung sehen, mehr für die Verkehrssicherheit zu tun.

Mehr Handlungsbereitschaft für die Sicherheit im Straßenverkehr wird auch von Seiten der Städte und Gemeinden (47\%), sowie von der Polizei (42\%) und dem Staat (38\%) gefordert. Zugleich werden mit $14 \%$ den Vereinen und Verbänden eine eher untergeordnete, jedoch nicht zu vernachlässigende Position bezüglich der Verkehrssicherheit eingeräumt. Auch sollten Autohersteller und Automobilindustrie mehr Initiative hinsichtlich der Verkehrssicherheit zeigen.

Tabelle 2:

Verantwortung für Verkehrssicherheit

\begin{tabular}{|l|c|c|}
\hline \multicolumn{1}{|c|}{ Verantwortlich für Verkehrssicherheit } & N & $\%$ \\
\hline die anderen Verkehrsteilnehmer & 888 & 53 \\
\hline ich selbst & 852 & 51 \\
\hline Städte und Gemeinden & 783 & 47 \\
\hline die Polizei & 711 & 42 \\
\hline der Staat & 642 & 38 \\
\hline Vereine und Verbände & 231 & 14 \\
\hline Autohersteller / Automobilindustrie & 12 & 1 \\
\hline Niemand & 16 & 1 \\
\hline n = 1.680
\end{tabular}




\section{Wie regelkonform verhalten sich Deutschlands Verkehrsteil- nehmer an Verkehrsampeln?}

\section{Fußgänger}

Das Übertreten einer roten Ampel ist bei Fußgängern ein weitverbreitetes Phänomen. Von insgesamt 514 Personen haben $5 \%$ in den letzten 12 Monaten oft oder sehr oft eine rote Ampel im Straßenverkehr übertreten. Weitere $14 \%$ missachteten gelegentlich rote Fußgängerampeln und $25 \%$ geben an, selten das Rotlicht missachtet zu haben. $53 \%$ der Befragten verhielt sich regelkonform. Zudem werden Rotlichtmissachtungen häufig bei anderen Fußgängern beobachtet. $50 \%$ der Befragten geben an, oft oder sehr oft Rotlichtmissachtungen anderer Fußgänger wahrzunehmen und $38 \%$ der Fußgänger beobachtet dies gelegentlich.

\section{Fahrradfahrer}

Von insgesamt 192 befragten Fahrradfahrern haben in den letzten 12 Monaten $1 \%$ oft eine rote Ampel missachtet, $17 \%$ taten dies gelegentlich. Weitere $27 \%$ haben im letzten Jahr selten eine rote Ampel passiert und 55\% taten dies nie. Knapp 10\% der Befragten geben an, häufig Rotlichtübertretungen von Fahrradfahrern zu beobachten und $50 \%$ beobachten dies gelegentlich.

\section{Pkw-Fahrer}

Von insgesamt 231 befragten Pkw-Fahrern geben $3 \%$ an, in den letzten 12 Monaten oft über eine rote Ampel gefahren zu sein. $27 \%$ missachteten das Rotlicht gelegentlich und nahezu die Hälfte (48\%) überfuhr selten eine rote Ampel. Lediglich $22 \%$ der Pkw-Fahrer geben an, nie rote Ampeln missachtet zu haben. Häufig werden Rotlichtmissachtungen bei anderen Pkw-Fahrern beobachtet. 37\% nehmen dies oft oder sehr oft bei anderen Pkw-Fahrern wahr und über die Hälfte (58\%) der Personen beobachtet eine Rotlichtmissachtung gelegentlich. Lediglich $5 \%$ haben selten oder sehr selten Rotlichtübertretungen bei anderen Fahrern bemerkt.

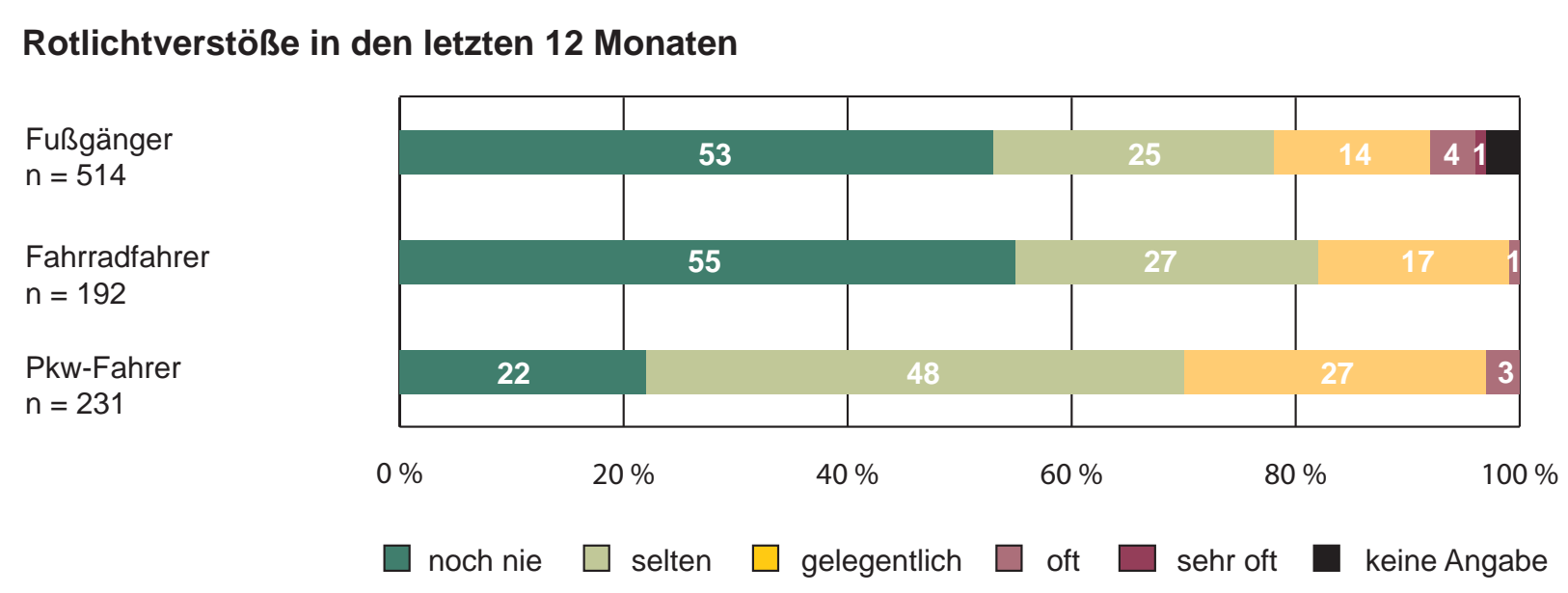

(C)UDV 2010

\section{Abbildung 7:}

Rotlichtverstöße in den letzten 12 Monaten 


\section{Sind Kontrolldichte und Strafen in Deutschland hoch genug?}

\section{Rotlichtübertretung:}

\section{Fußgänger}

Die Registrierung durch die Polizei halten 69 \% der Befragten für unwahrscheinlich. Nach der Strafe für Rotlichtübertretungen gefragt, liegt die Einschätzung mit $45 €, 1$ Punkt und 0,5 Monaten weit über dem tatsächlichen Strafmaß von $5 €$ Geldstrafe. Die tatsächliche Strafe von $5 €$ wird von der Mehrheit der Befragten (53\%) daher auch als nicht hart empfunden.

\section{Fahrradfahrer}

Die Sanktionierung von Rotlichtverstößen ist bei Fahrradfahrern selten. Nur $3 \%$ geben an, bisher für einen Rotlichtverstoß bestraft worden zu sein. Auch hält die Mehrheit (53\%) es für unwahrscheinlich, dafür von der Polizei registriert zu werden. Die Strafe für Rotlichtübertretungen für Radfahrer wird mit 137€, 2 Punkten und 1,3 Monaten Führerscheinentzug ebenfalls überschätzt. Die tatsächliche Strafe von $45 €$ und 1 Punkt empfand die Mehrheit der Probanden als kaum oder gar nicht hart und nur $32 \%$ als etwas hart.

\section{Autofahrer}

Die Sanktionierung von Rotlichtverstößen ist bei Pkw-Fahrern selten. Nur 7\% der Befragten geben an, in den letzten 12 Monaten für Rotlichtübertretungen bestraft worden zu sein. Auch gibt knapp die Hälfte der Befragten (49\%) an, dass es unwahrscheinlich ist, bei Rotlichtübertretungen von der Polizei registriert zu werden. Die tatsächliche Strafe von $90 €$ und 3 Punkten, die bei einer Rotlichtübertretung droht, wird von den befragten Pkw-Fahrer mit 118,73€, 1,8 Punkten und 0,6 Monaten Führerscheinentzug bei der Geldstrafe über und bei den Punkten unterschätzt. Eine derartige Strafe trifft etwa $70 \%$ der Pkw-Fahrer hart oder sehr hart und lediglich $9 \%$ würde eine solche Strafe gar nicht oder kaum treffen.

\section{Subjektive Entdeckungswahrscheinlichkeit von Rotlichtverstößen}

\section{Fußgänger}

$\mathrm{n}=514$

Fahrradfahrer

$\mathrm{n}=192$

Pkw-Fahrer

$\mathrm{n}=231$

(C) UDV 2010

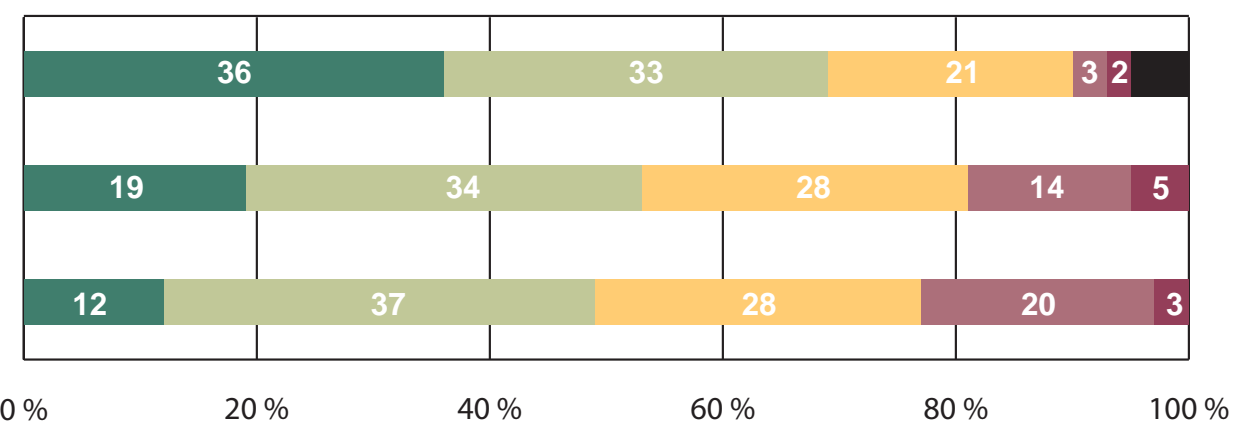

sehr unwahrscheinlich

eher unwahrscheinlich

teils / teils

eher wahrscheinlich 


\section{Geschwindigkeit:}

Die empfundene Kontrolldichte für Geschwindigkeitsverstöße ist nicht sehr hoch. Lediglich $17 \%$ der Pkw-Fahrer geben an, dass es wahrscheinlich oder sehr wahrscheinlich ist, von der Polizei beim Überschreiten der Geschwindigkeitsvorschrift registriert zu werden und $40 \%$ stimmten der Aussage teilweise zu. In den letzten 12 Monaten wurden die befragten Pkw-Fahrer im Durchschnitt 0,27 Mal wegen Geschwindigkeitsverstößen bestraft, $85 \%$ nicht, 11\% einmal und 4\% mehr als einmal. Auch überschätzen Pkw-Fahrer mit $31 €$ Geldstrafe und 0,2 Punkten die Strafe für Geschwindigkeitsübertretungen von $11-15 \mathrm{~km} / \mathrm{h}$ innerhalb geschlossener Ortschaften von $25 €$. Die Strafe von $25 €$ wird daher von knapp $75 \%$ der Fahrer als gar nicht, kaum oder etwas hart empfunden.

\section{Alkohol:}

Beim Fahren unter Alkohol geben $15 \%$ der Pkw-Fahrer an, dass es wahrscheinlich oder sehr wahrscheinlich ist, von der Polizei registriert zu werden und weitere $53 \%$ stimmen dieser Aussage teilweise zu. Mit 261€ Geldstrafe, 3 Punkten und 0,8 Monaten Führerscheinentzug unterschätzen Pkw-Fahrer die tatsächliche Strafe für einen über dem gesetzlichen Grenzwert gemessenen Blutalkoholwert von 500€, 4 Punkten und 1 Monat Führerscheinentzug. Von $48 \%$ der Fahrer wird diese Strafe als sehr hart und von weiteren $28 \%$ als hart empfunden.

\section{Subjektive Entdeckungswahrscheinlichkeit von Pkw-Fahrem für Geschwindigkeit und Alkohol}

Geschwindigkeit
$n=231$

Alkohol

$\mathrm{n}=204$

@ UDV 2010

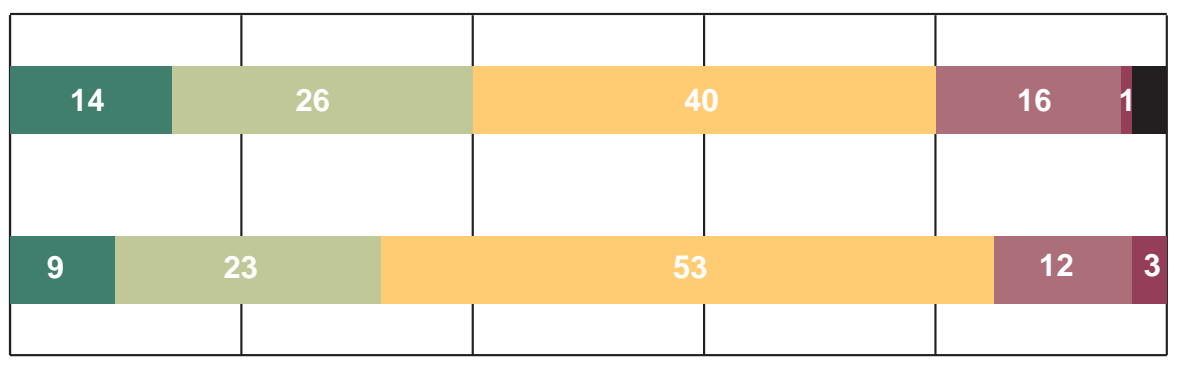

$0 \%$

$20 \%$

$40 \%$

$60 \%$

$80 \%$

$100 \%$

sehr unwahrscheinlich

$\square$ eher wahrscheinlich

eher unwahrscheinlich

teils / teils

sehr wahrscheinlich

Abbildung 9:

Subjektive Entdeckungswahrscheinlichkeit von Pkw-Fahrern für Geschwindigkeit und Alkohol 


\section{Wer sind Deutschlands Geländewagen (SUV)-Fahrer?}

Im Vergleich zu anderen Pkw-Fahrern unterscheiden sich SUV-Fahrer im Alter, Geschlecht und Einkommen. Im Durchschnitt sind SUV-Fahrer 45 Jahre alt und zu $58 \%$ männlich, Pkw-Fahrer hingegen im Durchschnitt 50 Jahre alt und zu $55 \%$ männlich. SUV-Fahrer verfügen zudem über ein durchschnittlich höheres Haushaltseinkommen als Pkw-Fahrer.

Anhand der Pkw-Verfügbarkeit und der Pkw-Nutzung lässt sich nachweisen, dass SUV-Fahrer ihren Pkw tendenziell öfter nutzen bzw. eine höhere Fahrleistung angeben als durchschnittliche Pkw-Fahrer. Im Mittel liegt die durchschnittliche jährliche Fahrleistung von SUV-Fahrern um etwa $3.788 \mathrm{~km}$ höher als bei Pkw-Fahrern.

SUV- und Pkw-Fahrer wurden bezüglich einer Reihe von Merkmalen des eigenen Verkehrsverhaltens und der wahrgenommenen Verkehrssicherheit verglichen. Nur in der Wahrnehmung der eigenen Verkehrssicherheit unterschieden sich beide Gruppen. So fühlen sich SUV-Fahrer im Vergleich zu Pkw-Fahrern sicherer im Straßenverkehr. Der Grund für das stärkere Sicherheitsgefühl von SUV-Fahrern wird in der häufigeren Pkw-Nutzung und höheren Fahrleistung gesehen. Denn alle Pkw-Fahrer fühlen sich unsicherer, je weniger häufig sie den Pkw nutzen. Beide Gruppen, SUV- und Pkw-Fahrer, fühlen sich am sichersten in Wohngebieten und am wenigsten sicher in Autobahnbaustellen. Auch bei der Frage, wo sich Pkw-Fahrer sicher fühlen, geben SUV Fahrer allgemein ein höheres Sicherheitsgefühl als Pkw-Fahrer an. Die Rangreihe der Orte ist aber bei beiden Gruppen gleich. So fühlen sich beide Gruppen am sichersten in Wohngebieten und am wenigsten sicher in Autobahnbaustellen.

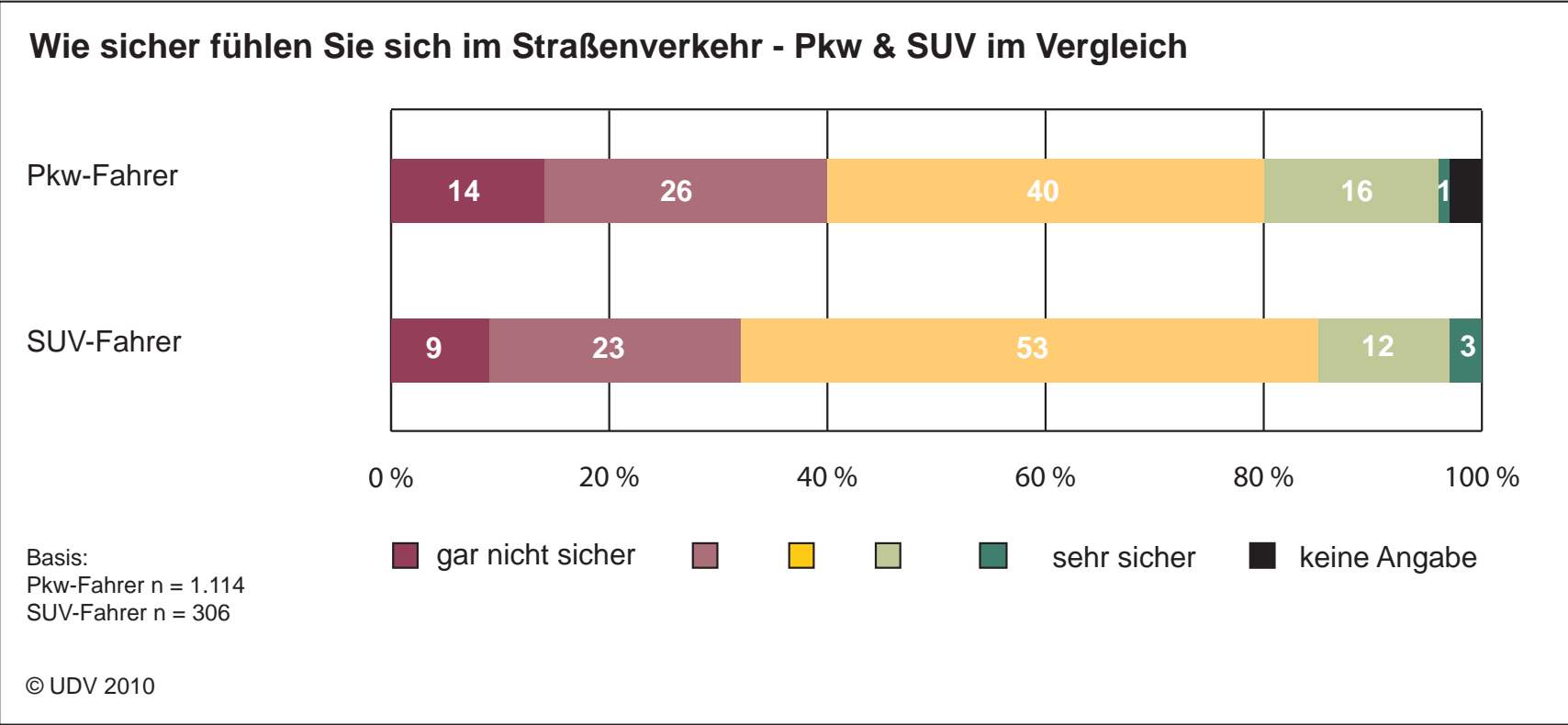

Abbildung 10:

Wie sicher fühlen sie sich im Straßenverkehr - Pkw \& SUV im Vergleich 


\section{Wie verhalten sich Deutschlands Autofahrer beim...}

\section{Telefonieren?}

Die Mehrheit der befragten Pkw-Fahrer empfindet das Telefonieren ohne Freisprechanlage als sehr risikoreich bzw. als risikoreich. Trotzdem geben $3 \%$ an, während der Autofahrt häufig oder sehr häufig ohne Freisprechanlage zu telefonieren und weitere $10 \%$ telefonieren teilweise ohne Freisprechanlage.

Als weniger riskant wird die Nutzung von Freisprechanlagen während der Fahrt empfunden, etwa ein Viertel empfinden die Nutzung solcher Anlagen während der Fahrt als teilweise risikoreich und $43 \%$ empfinden das Telefonieren mit Freisprechanlagen als gar nicht risikoreich bzw. nicht risikoreich. Mit solchen Anlagen telefonieren $12 \%$ der Pkw-Fahrer häufig oder sehr häufig. $60 \%$ nutzen nie Freisprechanlagen.

Zwischen Männern und Frauen gab es keine Unterschiede in der Häufigkeit oder der Risikowahrnehmung des Telefonierens mit oder ohne Freisprechanlage.

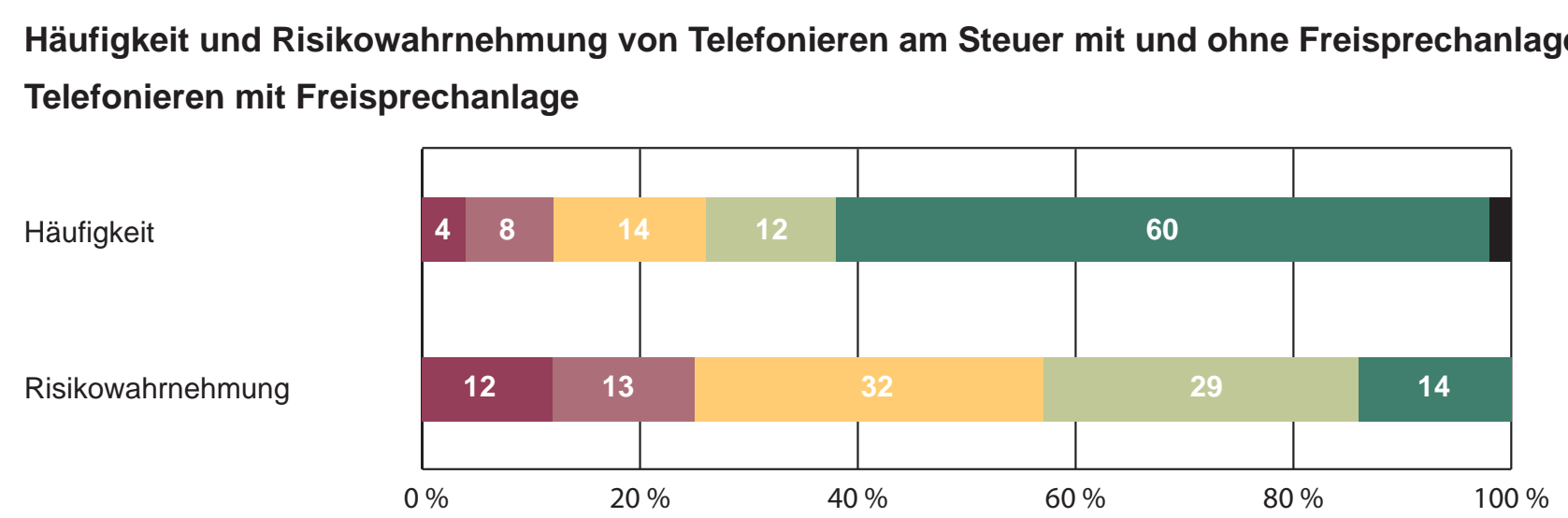

Telefonieren ohne Freisprechanlage

Häufigkeit

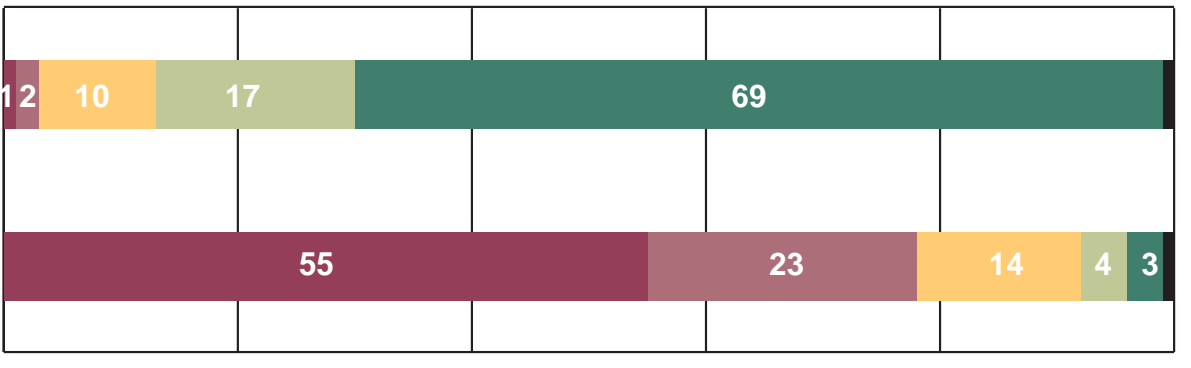

R isikowahrnehmung

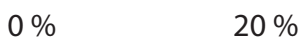

$40 \%$

$60 \%$

$80 \%$

$100 \%$

$n=1.420$

sehr häufig /sehr risikoreich

nie / gar nicht risikoreich

keine Angabe

(๑) UDV 2010

Abbildung 11:

Häufigkeit und Risikowahrnehmung von Telefonieren am Steuer mit und ohne Freisprechanlage 


\section{SMS schreiben?}

Vier von fünf Pkw-Fahrern schreiben nie SMS während der Fahrt und $5 \%$ schreiben selten eine SMS. Die Zahl der Personen, die sehr häufig oder häufig SMS während des Autofahrens schreiben beläuft, sich auf etwa $3 \%$.

Die meisten Personen (87\%) schätzen das Risiko, welches mit dem Verfassen einer SMS einhergeht, als sehr hoch oder hoch ein. Nur ein geringer Teil geht von einem geringen oder keinem Risiko aus (3\%). Nachgewiesen werden konnte ein statistisch signifikanter Zusammenhang zwischen der Häufigkeit und der Risikowahrnehmung des SMS Schreibens während der Fahrt. Personen, die das Verfassen von SMS als weniger risikoreich einstufen, schreiben auch häufiger SMS während der Fahrt.

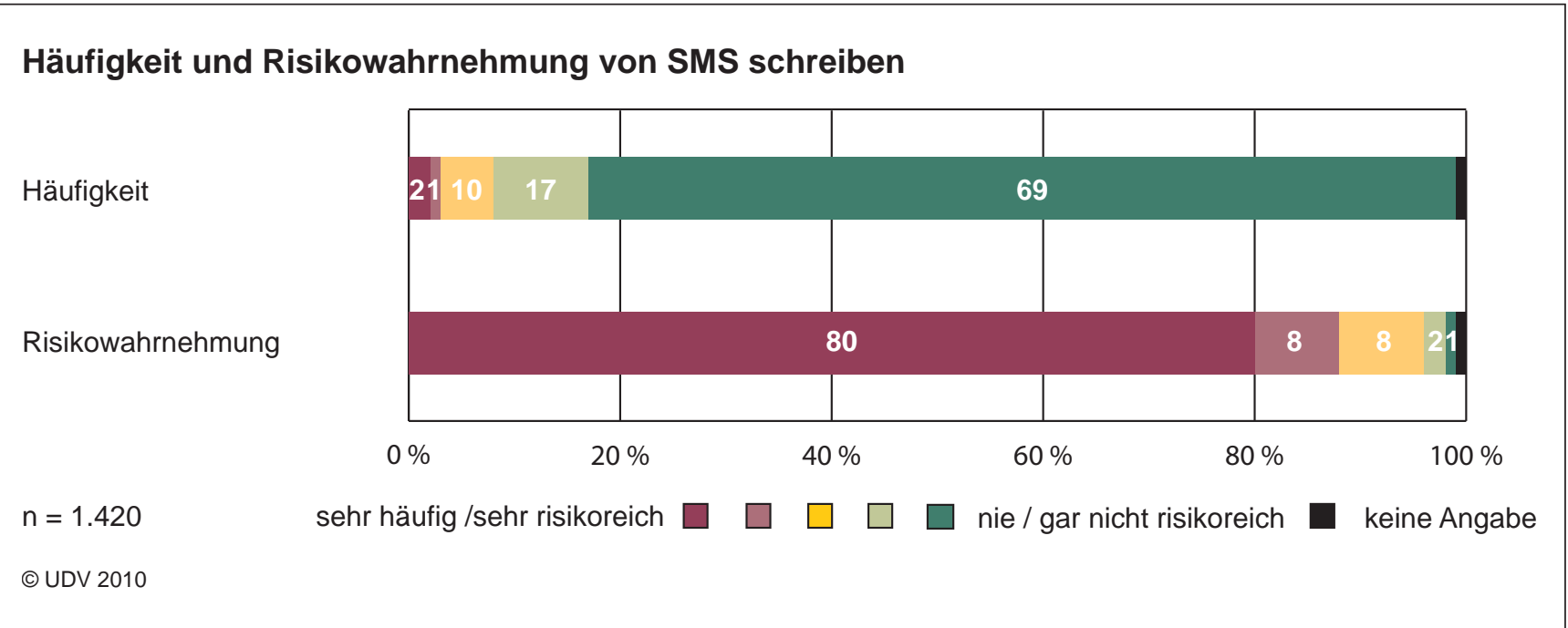

Abbildung 12:

Häufigkeit und Risikowahrnehmung vom SMS schreiben 


\section{Wie sicherheitsbewusst sind Deutschlands Autokäufer?}

Die Sicherheit eines Autos spielt neben der Zuverlässigkeit (93\%) und der Wirtschaftlichkeit (84\%) eine wichtige Rolle für die Kaufentscheidung. Insgesamt nennen 87\% der Pkw-Fahrer die Sicherheit als wichtiges oder sehr wichtiges Kriterium für die Auswahl ihres Fahrzeuges.

Die Bewertung nach EuroNCAP (European New Car Assessment Programme), die herstellerunabhängige Anhaltspunkte für die Sicherheit eines Fahrzeuges bietet, nennen lediglich $34 \%$ als wichtiges oder sehr wichtiges Entscheidungskriterium.

\section{Kriterien für den Kauf eines Fahrzeuges}

Wie wichtig sind Ihnen beim Kauf Ihres Fahrzeuges...

Zuverlässigkeit

Sicherheit

Wirtschaftlichkeit

Umweltverträglichkeit

Design

Marke

Bewertung nach EuroNCAP

hohe Motorleistung

Basis:

Pkw-Fahrer $2010 \mathrm{n}=1.420$

(c) UDV 2010

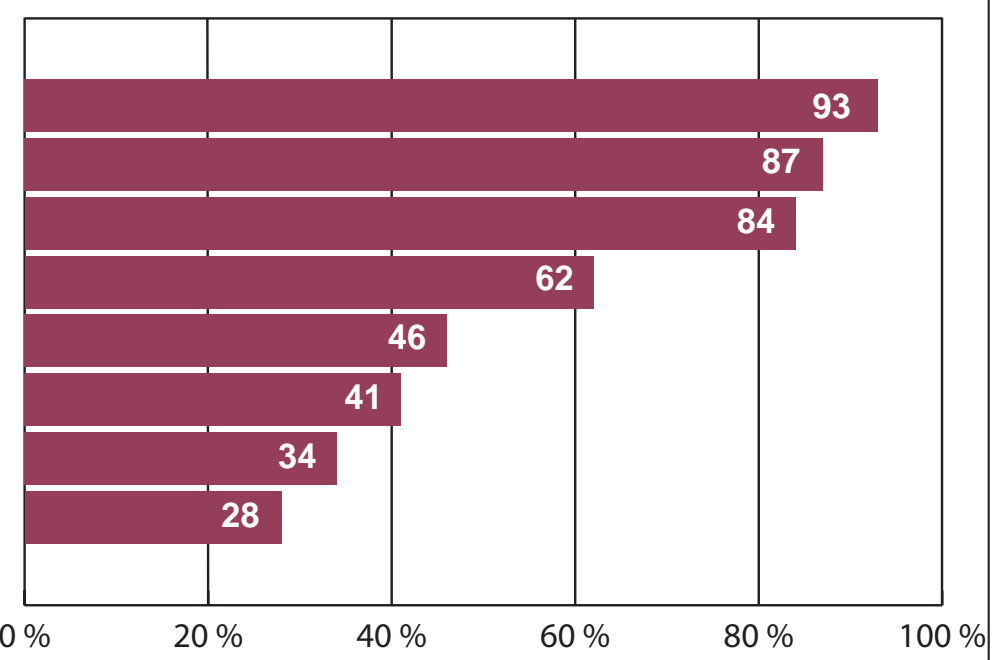

Zustimmung zu den Kategorien „wichtig“ und „sehr wichtig“

Abbildung 13:

Kriterien für den Kauf eines Fahrzeuges

Statistisch signifikante Unterschiede in der Bewertung der Kriterien für den Fahrzeugkauf gibt es beim Alter, Geschlecht und der Pkw-Nutzung. So messen Frauen der Sicherheit, Wirtschaftlichkeit und Umweltverträglichkeit eine größere Wichtigkeit zu als einer hohen Motorleistung. Ältere Pkw-Fahrer bewerten die Zuverlässigkeit, Sicherheit, Wirtschaftlichkeit und Umweltverträglichkeit wichtiger als jüngere Pkw-Fahrer und Vielfahrer halten Sicherheit und Umweltverträglichkeit für tendenziell wichtiger als Wenigfahrer. 


\section{Wie gut kennen deutsche Autofahrer Fahrerassistenzsysteme?}

Am bekanntesten ist das Elektronische Stabilitätsprogramm. 90\% der befragten Pkw-Fahrer kennen dieses System. Notbremsassistent, Spurverlassens- und Totwinkelwarner sind ebenfalls einer Mehrheit der Befragten (etwa $65 \%$ ) bekannt.

Am häufigsten ausgestattet sind die Pkw der Befragten mit ESP. 50\% der Pkw-Fahrer geben an, dass ihr Pkw mit diesem System versehen ist. Lediglich $14 \%$ der Pkw sind mit einem Notbremsassistent ausgestattet. Spurverlassenswarner oder Totwinkelwarner sind dagegen kaum vorhanden.

Männer kennen den Notbremsassistent, den Spurverlassenswarner und den Totwinkelassistent signifikant häufiger als Frauen. Auch kennen jüngere Pkw-Fahrer ESP und Spurverlassenswarner häufiger als ältere Pkw-Fahrer. Vielfahrer kennen den Notbremsassistent, Spurverlassenswarner und Totwinkelwarner häufiger als Wenigfahrer. Ebenso ist deren Pkw häufiger mit ESP ausgestattet als Pkw von Wenigfahrern.

\section{Bekanntheit und Verfügbarkeit von FAS}

Elektronisches

Stabilitätsprogramm

Notbremsassistent

Spurverlassenswarner

Totwinkelassistent

Basis:

P kw-Fahrer $n=1.420$

๑) UDV 2010

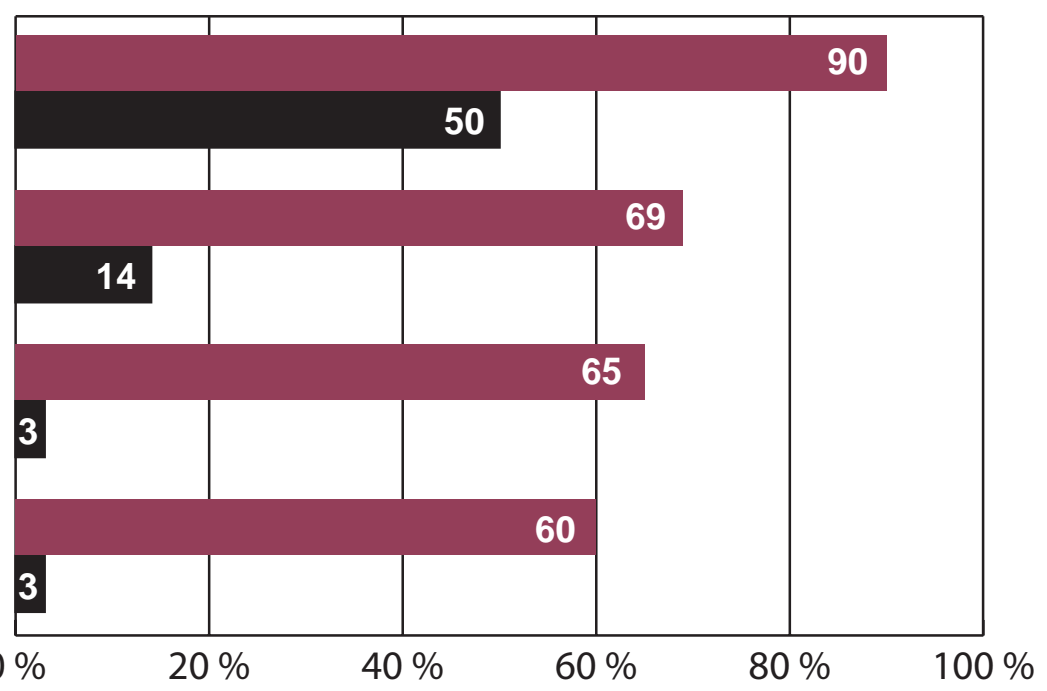

Bekanntheit
Verfügbarkeit 


\section{GDV}

DIE DEUTSCHEN VERSICHERER

Gesamtverband der Deutschen Versicherungswirtschaft e. V.

Wilhelmstraße 43/43G, 10117 Berlin

Postfach 0802 64, 10002 Berlin

Telefon 030/20 20 - 50 00, Fax 030/20 20 - 6000

www. gdv.de, www.udv.de 